\title{
A Proteomic Profile of Cervical Remodeling in Mice during Early and Late Pregnancy
}

John Schwabe ${ }^{1}$, Siobhan M Donnelly ${ }^{1}$, Subrina Jesmin ${ }^{2}$, Phyllis Leppert ${ }^{3}$ and Chishimba Nathan Mowa ${ }^{1,4 *}$

${ }^{1}$ Appalachian State University, Department of Biology, Boone, NC, USA

${ }^{2}$ Tsukuba University, Department of Internal Medicine, Tsukuba, Japan

${ }^{3}$ Duke School of Medicine, Department of Obstetrics and Gynecology, Durham, NC, USA

${ }^{4}$ University of Johannesburg, Faculty of Health Sciences, Department of Anatomy and Physiology, Johannesburg, South Africa

\begin{abstract}
Objective: Decipher mechanisms that underlie cervical remodeling $(\mathrm{CR})$ during pregnancy.

Design and population: Examine the proteomic profile of mice cervices in early and late pregnancy and verify data generated using Western Blot and real time PCR analysis.
\end{abstract}

Main outcome measures: Identify signature proteins.

Results: In total, seventy three (73) signature proteins were identified and were found up regulated in pregnancy, namely proteins associated with: a) collagen presence and organization (4), notably biglycan and lumican, b) immune scavenging functions and c) cytoskeletal activity. These proteomic trends were verified through Western blot and real time PCR analysis.

Conclusion: These signature proteins and their expression profile collectively suggest a complex biological process involving extracellular matrix (ECM)- and immune -associated factors. Based on these data, we propose that biglycan may provide the link between natural birth and inflammation-mediated pre-term birth.

\section{Keywords: Proteomics; Cervix; Mice; Pregnancy}

\section{Introduction}

The cervix undergoes highly complex biochemical and mechanical changes during and immediately after pregnancy [1]. This multifactorial process, termed cervical remodeling (CR), is divided into four distinct but overlapping stages, i.e., cervical softening, ripening, dilation and repair [2,3]. In mice, CR begins at about day 12 of a 19-day gestation, whereas in human it begins in the first trimester of pregnancy [2]. The CR alterations completely transforms the cervix from a non-pliable rigid structure to a flexible one, thus enabling it to perform its key role, notably, the retention of the rapidly growing fetus in uteri, while simultaneously preparing for its final passage at birth [1]. Unfortunately, the exact mechanisms that triggers and regulate these alterations are currently poorly understood. A comprehensive knowledge of this process (CR) is essential in managing obstetrical complications, such as preterm labor and cervical lacerations, which have serious consequences for the health of the fetus, as well as the mother [4,5]. Because of the complex nature of CR and involvement of multiple factors, which have in-built redundancies, there is need to use a variety of techniques that vary in scope and sensitivity, such as proteome-wide techniques in combination or addition to the reductionist approaches that focus on a single gene or protein at a given time, which has been the predominant and historical approach. Such robust techniques could shed more light on this complex process and perhaps help resolve the current debates on the two competing models for term-birth $[6,7]$.

In the early 1980s, Mont Liggins proposed a model that inflammatory cells induce changes in the cervical extracellular matrix that lead to cervical ripening [8], although the proposed mechanism as a model for all parturition was questioned earlier on, suggesting that preterm and term had differing biochemical triggers $[9,10]$. The Liggins model accounts for the increased incidence of preterm delivery in women with inflammatory diseases $[5,11]$ and has been the dominant model, until very recently [12-14]. While the Liggins model certainly explains the increased prevalence of preterm birth in women with inflammatory diseases, such as chorioamnionitis, only less than half of all pre-term births can be linked to an inflammatory disease or condition [11]. More recent studies support the idea that while inflammation may cause parturition in certain circumstances, it (inflammation) is not involved in natural physiologic term birth, as proposed by the Liggins model [15]. For example, complete elimination of neutrophils and partial elimination of macrophages in mice does not block natural term birth [16]. Further, it has been shown that WBC infiltration, as well as the subsequent expression of pro-inflammatory factors in the cervix peak after, rather than, during pregnancy and that inconsistent or inaccurate tissue collection times in previous studies may have led to erroneous conclusions [16]. In light of these recent data, a new model has been proposed that places cervical epithelial cells as the initiator and mediator of cervical remodeling [15]. The new model suggest that the cervical epithelia secrete factors that cause slow structural changes in the maturity and organization of fibrillar collagen in the cervix and that decreased secretion of lysyl oxidase (LOX), the enzyme that crosslinks collagen molecules, leads to the softening of the cervix [17]. Fewer cross-links between collagen fibrils lead to fibrils of greater diameter, which increase the compliance of the tissue $[10,11]$.

Here, we use proteomics, a proteome-wide technique, to identify

*Corresponding author: Chishimba Nathan Mowa, Appalachian State University, Department of Biology, Rankin Science North Building N219, 572 River Street, Boone, NC 28608, USA, Tel: +1-828-262 2676; Fax: +1-828-262 2127; E-mail: mowacn@appstate.edu

Received September 04, 2013; Accepted December 30, 2013; Published January 10, 2014

Citation: Schwabe J, Donnelly SM, Jesmin S, Leppert P, Mowa CN (2014) A Proteomic Profile of Cervical Remodeling in Mice during Early and Late Pregnancy. J Steroids Horm Sci 5: 123. doi:10.4172/2157-7536.1000123

Copyright: (c) 2014 Schwabe J, et al. This is an open-access article distributed under the terms of the Creative Commons Attribution License, which permits unrestricted use, distribution, and reproduction in any medium, provided the original author and source are credited. 
the signature proteins expressed in mice during early and late pregnancy. The generated proteomic data was verified by Western blot and real time PCR analysis. These findings point to further refinement of the concept that the cervical epithelial cells contribute to cervical remodeling and suggest a role for mechanical signaling in this process.

\section{Materials and Methods}

\section{Animals used, surgeries and tissue harvest}

Pregnant (day 11 and 17) and non-pregnant mice sourced from Charles Rivers (C57BL6/129SvEv), were used in these studies $(n=3$ 5 ), as specified below. Ovariectomy was performed on non-pregnant animals in order to delineate proteins that are expressed in the absence of ovarian sex steroid hormones, thus subtracting the confounding effects of indigenous ovarian sex steroid hormones. Prior to tissue harvest, animals were euthanized by a lethal injection of Sodium Pentobarbital (Sleepaway ${ }^{\circledR}$, Fort Dodge Laboratories Inc., Burlingame, CA) and perfused intra-cardially to flash out blood. Cervical tissues were carefully harvested under a stereomicroscope in order to exclude vagina and uterine tissues as much as is practical; processed and analyzed using proteomics analysis and the generated proteomics data were then verified using real time polymerase chain reaction (real time PCR' $n=5$ ) and Western blot analysis $(n=5)$. Specifically, non-pregnant ovariectomized (removal of ovaries) mice, as well as mice during day 11 (early pregnancy) and day 17 (late pregnancy) of gestation were utilized. Surgeries (ovariectomy) for non-pregnant animals were performed as previously described $[18,19]$. All non-pregnant animals with normal uterine size, seven days after ovariectomy, were eliminated from the study. All experiments were performed in accordance with the Guide for the Care and Use of Laboratory Animals of the local institution (Appalachian State University) and the NIH guidelines (NIH publication number 86-23), and efforts were made to minimize both animal suffering and the number of animals used.

\section{Techniques used}

Protein expression studies: Proteomics and Western blot Analysis. All the key proteins (proteomics) expressed during the three selected physiological conditions (non-pregnant, early and late pregnancy) and two selected signature proteins from proteomics data (Western blot) were studied using proteomics and Western blot analysis, respectively, as described below. Tissues from the non-pregnant ovariectomized animals were used as negative control for baseline data.

General protein extraction (proteomics and western blot analysis): Total protein for proteomics and Western blot were extracted from whole cervical tissues using the protein inhibitor cocktail and cell lysis buffer from Sigma Aldrich (St Louis) and the supernatant was divided into half and immediately stored at $-80^{\circ} \mathrm{C}$. The core facility Laboratory of the David Murdock Research Institute (Kannapolis, NC) performed the proteomics analysis and selected proteins from the proteomics data were then verified by Western blot and real time PCR, described later below.

\section{Proteomics:}

Sample preparation: The samples were assayed using the Thermo Scientific Micro bicinchoninic acid (BCA) Protein Assay kit to determine protein concentration. The samples were diluted to fall within the linear working range of the kit $(5-200 \mu \mathrm{g} / \mathrm{mL})$ and the concentrations were calculated based on absorbance values compared to a bovine serum albumin (BSA) standard curve. The samples were filtered using a $3 \mathrm{kD}$ ultra centrifugal filter to exchange the dissolution solvent with a mass spectrometry friendly buffer. $100 \mu \mathrm{L}$ of sample was diluted to $500 \mu \mathrm{L}$ with $50 \mathrm{mM}$ ammonium bicarbonate and filtered. The samples viewed in Scaffold (Proteome Software, Portland, OR). QC and study samples were evaluated to confirm data quality. Liquid chromatography total ion current (TIC) outputs were assessed for signal quality and changes in signal intensity. Results were also monitored for signal trends, such as a consistent increase or decrease in TIC maximum values, and MASCOT search results were used to monitor the quality of the mass spectrometry (MS) data (Table 1).

Western blot analysis: Quantitative analysis of protein expression for the following 2 selected signature proteins from the proteomics data was performed to verify and supplement proteomics analysis, as described previously $(18,19)$ : a) Laminin $\gamma-1$ and b) Calcium-Activated Chloride Channel Regulator-1 (CLCA1). Membranes were stained with specific primary antibodies at 1:500 dilution, washed several time prior to incubation with the secondary antibody, i.e., Donkey anti-rabbit IgG HRP conjugated and Streptactin HRP antibodies for $1 \mathrm{~h}$ at RT, and then washed again before incubating them with luminescenceproducing enzyme, namely limunol-enchancer/peroxidase solution and developed. Images were analyzed with ImageJ program (NIH) The specific bands of the proteins of interest were identified using a standard ladder with known molecular weight.

Gene expression study: real time PCR: Gene expression analysis was performed using real time PCR to verify data generated by proteomics analysis, as described previously [18,19]. Specifically, based on the proteomics data, the mRNA expression of the following genes were

\begin{tabular}{|c|c|c|}
\hline System & Parameter & Setting \\
\hline \multirow[t]{2}{*}{ Autosampler } & Temperature & $4^{\circ} \mathrm{C}$ \\
\hline & Injection & $5.0 \mu \mathrm{L}$ prepared sample \\
\hline \multirow[t]{2}{*}{ LC Trap Column } & Sample load time & $5 \mathrm{~min}$ \\
\hline & Trap flow rate & $5 \mu \mathrm{L} / \mathrm{min}$ \\
\hline \multirow[t]{8}{*}{ LC Analytical Column } & Column temperature & $55^{\circ} \mathrm{C}$ \\
\hline & Flow rate & $0.4 \mu \mathrm{L} / \mathrm{min}$ \\
\hline & Gradient & Initial conditions: $98 \% \mathrm{~A}$ \\
\hline & & $t=60 \min , 60 \% A$ \\
\hline & & $t=65 \min , 5 \% A$ \\
\hline & & $t=70 \min , 5 \% A$ \\
\hline & & $t=75 \min , 98 \% A$ \\
\hline & & $t=95 \min , 98 \% A$ \\
\hline \multirow{17}{*}{$\begin{array}{l}\text { LTQ Orbitrap XL Mass } \\
\text { Spectrometer }\end{array}$} & Experiment type & Nth Order Double Play \\
\hline & \# Dependent scans & 5 \\
\hline & Source voltage & $2.0 \mathrm{kV}$ \\
\hline & Capillary temperature & $200^{\circ} \mathrm{C}$ \\
\hline & Source current & $1.0 \mu \mathrm{A}$ \\
\hline & Capillary voltage & $47.0 \mathrm{~V}$ \\
\hline & Tube lens & $100.0 \mathrm{~V}$ \\
\hline & FTMS survey scan time & $1.0 \mathrm{sec}$ \\
\hline & FTMS resolution & 60,000 \\
\hline & Survey time & $90 \mathrm{~min}$ \\
\hline & Survey ion mode & ES Mode \\
\hline & Survey polarity & Positive \\
\hline & Survey mass range & $375-2000$ \\
\hline & Activation type & CID \\
\hline & Normalized collision energy & 35.0 \\
\hline & ITMS MS/MS mass range & $50-2000$ \\
\hline & ITMS MS/MS scan time & $0.2 \mathrm{sec}$ \\
\hline
\end{tabular}

Table 1: $L C$ and MS Instrument Parameters used in the study: Analytical samples were analyzed on a Thermo Scientific LTQ Orbitrap XL mass spectrometry system coupled to a Waters nano ACQUITY UPLC system using parameters presented in the Table below. 
selected and examined using real were rinsed with $200 \mu \mathrm{L}$ ammonium bicarbonate and filtered. The tubes were inverted and centrifuged to collect the sample. A volume of each sample corresponding to $35 \mu \mathrm{g}$ of protein (based on the protein quantization results) was used. The sample volumes were made equal by adding $50 \mathrm{mM}$ ammonium bicarbonate (AmBic) to a volume of $29.8 \mu \mathrm{L}$. A $1 \%$ solution of Rapigest was added to each sample to denature the proteins and the mixture was placed in a shaking heated mixer at $40^{\circ} \mathrm{C}$ for 10 minutes. Disulfide bonds were reduced by adding $200 \mathrm{mM}$ dithiothreitol (DTT) to each sample and the tubes were heated to $80 \mathrm{oC}$ for 15 minutes. Free sulfur atoms were alkylated with $400 \mathrm{mM}$ Iodoacetamide (IA) by placing the tubes in the dark for 30 minutes at room temperature (RT). A tryptic digest was performed by adding $0.7 \mu \mathrm{g}$ Gold-Mass Spectrometry grade trypsin to each tube and incubated overnight at $37^{\circ} \mathrm{C}$. Alcohol dehydrogenase $(\mathrm{ADH})$ digest from yeast was added to a final concentration of $50 \mathrm{fmol} /$ $\mu \mathrm{g}$ protein. The trypsin reaction was stopped and the Rapigest was degraded with the addition of $10 \%$ TFA $/ 20 \%$ acetonitrile/ $70 \%$ water that was then heated to $60^{\circ} \mathrm{C}$ for $2 \mathrm{~h}$. The samples were centrifuged and the supernatant pipetted into auto-sampler vials.

A protein standard (BSA) was carried through the reduction/ alkylation and digestion steps and used as a quality control (QC) of the sample preparation steps and instrument performance. Liquid chromatography (LC) solvents for peptide separation included: a) water containing $0.1 \%$ formic acid; and b) acetonitrile containing $0.1 \%$ formic acid. The study sequence consisted of the study sample injections bracketed by a pair of QC injections. Data from all study samples were acquired using Data Dependent ${ }^{\mathrm{TM}}$ scans (Nth order double play) on the LTQ Orbitrap XL. Database searches were performed in Elucidator (Rosetta Biosoftware) using MASCOT (Matrix Sciences, London, UK). Analytical results were also time PCR: a) Anterior Gradient Homolog-2; b) Calcium-Activated Chloride Channel Regulator-1 (CLCA1); c) Collagen $6 \alpha 3$ (COL6A3) and d) Laminin $\gamma$-1, using the same mice, i.e., strain and physiological conditions, described earlier. Gene expression analysis was performed in three steps, as described below.

\section{Statistical analysis}

Proteomics: Raw MS data files for the study samples, collected on the Thermo Orbitrap XL system, were processed in Elucidator. MS data were grouped in Elucidator based on sample group and alignment (Table 1). Sample groups were used to assist in data alignment, feature identification, and were utilized for QC assessment and group comparisons. Data were processed from retention time, 8-90 min. Thermo Orbitrap data files were searched using the Mascot search engine against the SwissProt mouse database (appended with yeast $\mathrm{ADH}$, March 02, 2010). The aligned mass features were annotated with these database search results using the results from the system Peptide Tellers and a predicted error rate of $1 \%$. MS data were summarized to the feature level, normalized, and an error-weighted ANOVA test was performed to compare the expression results between sample groups. Candidate differentially expressed markers were determined based on a $p<0.01$. Features were summarized by protein based on the results of the database search. Western blot and real time PCR: Data for Western

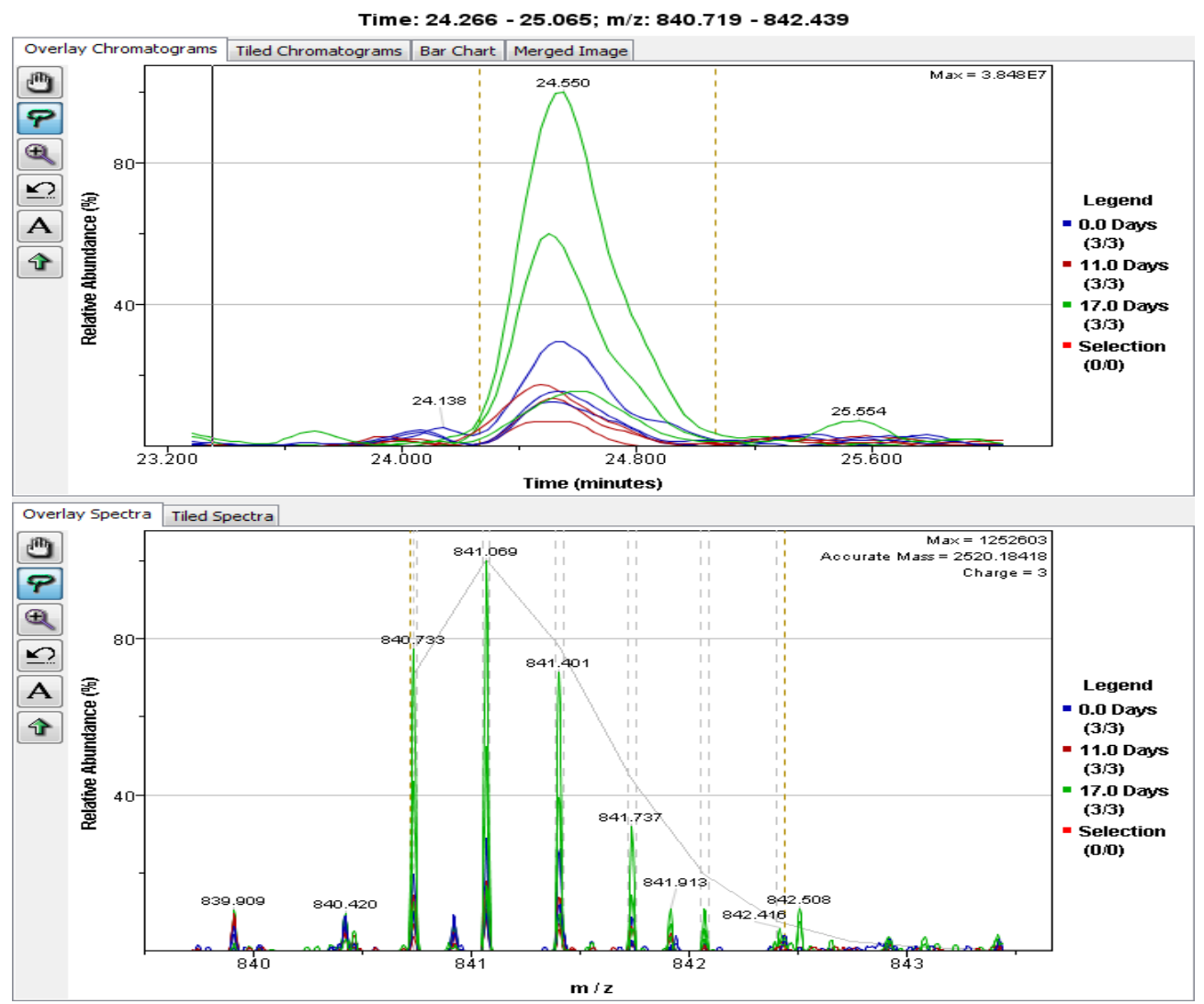

Figure 1: An Example of Aligned Mass Data: An example of aligned peptide signals for the data set is presented here: a) Sample labels: Figures are color-coded according to sample group. Selection (red) references an option to manually select a single sample to be highlighted. This option was not selected; b) Top pane/: Extracted ion chromatogram for $\mathrm{m} / \mathrm{z}$ 840.719-842.439 from retention time 24.266-25.065 minutes demonstrates retention time alignment across all samples for this signal; c) Bottom panel: Aligned masses in the mass range of $840.733-842.416$ for all samples in the study set. 
blot and real time PCR analyses were analyzed using Student's t test and ANOVA, followed by Scheffe's F-test for multiple comparisons. $P$-values of $<0.05$ were considered to be statistically significant.

\section{Results}

\section{Protein expression studies}

\section{Proteomics studies:}

Two hundred and six (206) proteins were identified in early and late pregnancy: Using the Rosetta Elucidator (Figure 1), a total of 206 distinct proteins was identified in cervical tissues of ovariectomized non-pregnant and pregnant mice (early and late pregnancy) (Figure 2). Of these 206 identified proteins, 73 were variably-expressed proteins in the cervix (Figures 3-7). As for protein names and intensities), of which 15 proteins (out of 73 proteins) were up regulated during late pregnancy, relative to early pregnancy and baseline; 23 proteins were up regulated during early pregnancy, relative to late pregnancy and baseline (non-pregnant mice), while 35 proteins were up regulated in cervix from the non-pregnant mice relative that of the pregnant mice (Appendix A for complete list of each variably expressed protein, their gene name, intensities, errors and fold changes) $(\mathrm{p}<0.05)$.

Proteins from diverse biological themes were variably expressed during pregnancy: Each of the variably expressed proteins was placed into a category based on its functions (Table 2). Biological categories were adapted from our previous DNA microarray publication [19]. Specifically, the following biological themes or processes (a-f) were up regulated in the cervix during pregnancy: a) protein kinase activity, b) proliferation, c) calcium binding, d) nucleus-related proteins, e) cytoskeleton-related proteins, f) extracellular matrix proteins (Table 2 ), whereas, in contrast (g-i), proteins associated with g) tissue remodeling, h) circulation, and i) carbohydrate metabolism were found to be down regulated during pregnancy (Table 2) and, on the other hand, $\mathrm{j}$ ) immune-related proteins and $\mathrm{k}$ ) cellular adhesion proteins displayed unclear trends of expression (Table 2, see Appendix B for fold change associated with each specific protein). It is important to note that some of the proteins identified by the proteomics analysis do not have known functions and could not be placed into a specific group (Supplementary).

Specific expression patterns of selected proteins: Expression of specific selected proteins were found to be significantly up or down regulated in the cervix during pregnancy. Specifically, these proteins include: 1) Laminin, with a fold change of 147 between baseline and early pregnancy and a total fold change of 139 between baseline and late pregnancy (Figure 3);2) calcium-activated chloride channel regulator, with a fold change of -58 between baseline and late pregnancy (Figure 4); 3) anterior gradient protein 2, with a fold change of 999 between baseline (non-pregnant) and early pregnancy (Figure 5); and 4) indolethamine $\mathrm{N}$-methyltransferase, with a fold change of 56 between baseline and late pregnancy (Figure 7B). Other target proteins included: a) collagen VI, with a fold change of 1.05 between baseline and early pregnancy and a total fold change of 15.8 between baseline and late pregnancy (Figure 6A); b) biglycan, with a fold change of 2.4 between baseline and early pregnancy and a fold change of 30.8 between baseline and late pregnancy (Figure 7A), c) lumican, with a fold change of -1.8 between baseline and early pregnancy and a total fold change of -1.8 between baseline and late pregnancy (Figure $7 \mathrm{C}$ ); and d) vinculin, with a fold change of -1.8 between baseline and early pregnancy and a fold change of -1.2 between baseline and late pregnancy (Figure 7D).

\section{Western blot and Real time PCR studies:}

Laminin mRNA and protein expressions are pronounced during late pregnancy in mice cervix: Data generated from both Western blot and real time PCR for expression of laminin mRNA and protein in mice cervix indicate pronounced expression during late pregnancy compared to early pregnancy and non-pregnant mice, thus verifying the proteomic data (Figure 3). However, there was a significant difference in the intensity of expression for Western blot and real time PCR, compared to proteomics (Figures 3B and 3C). In contrast, for proteomics, differences in intensity between late pregnancy and early pregnancy/non-pregnancy states were in million fold, whereas they were only 2.5 and 1.67 fold more in late pregnancy compared to early pregnancy and non- pregnant mice, for Western blot and real time PCR, respectively (Figure 3).

Levels of calcium-activated chloride channel regulator mRNA and protein rise during late pregnancy in mice cervix: Similar to expression of laminin mRNA and protein, the levels of calciumactivated chloride channel regulator $\mathrm{mRNA}$ and protein in late pregnancy were greater than early pregnancy and the non-pregnant mice, as revealed by both Western blot and real time PCR analysis

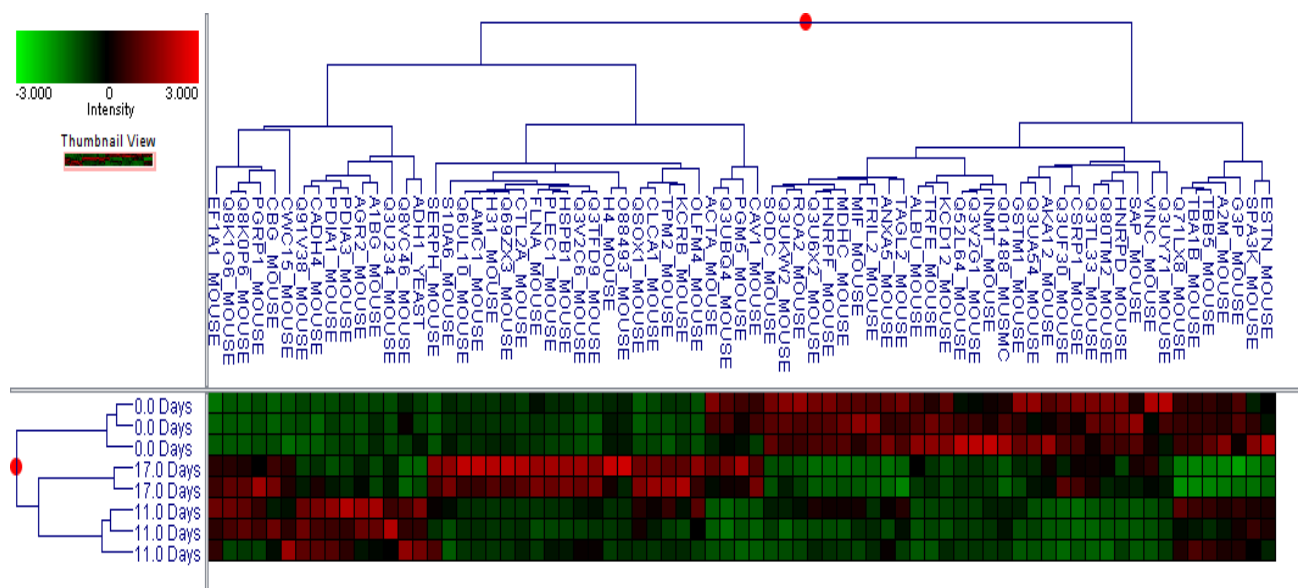

Figure 2: A distinct genome-wide protein expression profile in cervix of non-pregnant and pregnant mice: Cluster analysis reveal differentially expressed proteins of non-pregnant (Day 0), early pregnancy (Day 11) and late pregnancy mice cervices (Day 17), as determined by proteomics and analyzed by ANOVA analysis $(n=3, p=0.05)$ 
A

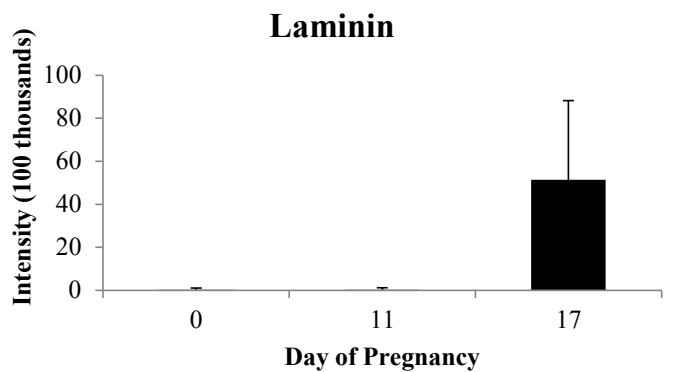

B

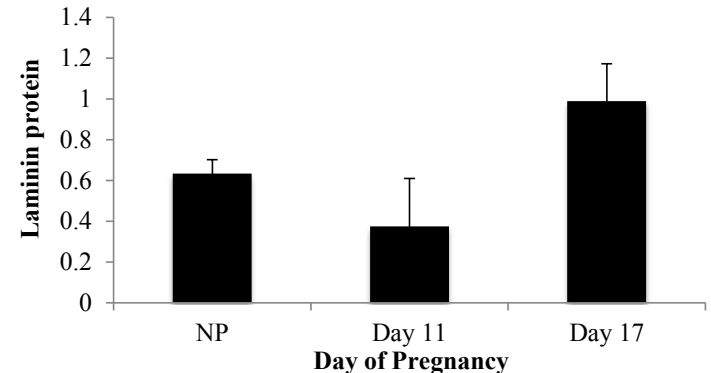

C

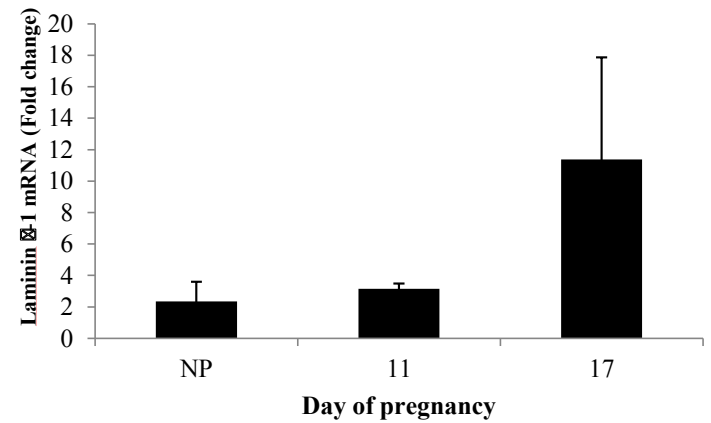

Figure 3: Profile of laminin expression in the mice cervix during pregnancy, as revealed by proteomics $(A)$, and verified by Western blot $(B)$ and real time PCR (C). Overall, all the three techniques demonstrated increase in levels of laminin during late pregnancy compared to non- and early-pregnancy. ( $n=3$ and 5 for proteomic and Western blot/real time PCR analyses, respectively; $p<0.05$ for $\mathrm{NP} /$ day 11 vs. day 17)

(Figure 4B and 4C), thus again verifying the proteomic data (Figures $4 \mathrm{~A})$. However, as was the case with laminin, there was a significant difference in the intensity of expression for Western blot and real time PCR (Figure 4B and 4C) compared to proteomics (Figure 4A). Differences in expression for proteomics between late pregnancy and early pregnancy/non-pregnancy states were tens of million folds, but only 2.5 and 1.67 fold for Western blot and about 3.0 and 1.5 folds for real time PCR more in late pregnancy than early pregnancy and nonpregnant mice, respectively (Figure $4 \mathrm{~B}$ and $\mathrm{C}$ ).

Anterior gradient protein 2 mRNA and protein expressions are up regulated in mice cervix in pregnancy: Overall, the expression of anterior gradient protein $2 \mathrm{mRNA}$ and protein in mice cervix were found to be up regulated between gestational days 11 and 17 but not in non-pregnant mice, as revealed by proteomics and real time PCR (Figure 5). However, transcription levels in early pregnancy were lower than those of late pregnancy (Figure 5). No Western blot analysis was performed in this particular study.

Expressions of collagen type VI, alpha $3 \mathrm{mRNA}$ and protein significantly increase in mice cervix during pregnancy: Generally, levels of collagen type VI, alpha 3 mRNA in mice cervix during pregnancy increased sharply compared to non-pregnant mice by as much as about 40-110 fold, as revealed by real time PCR (Figure 6). This was the sharpest rise of transcription in all the studied genes. However, levels of transcription during early pregnancy were about less than half of late pregnancy (Figure 6B). Overall, both real time PCR and proteomics data showed trends of increased expressions of protein and mRNA during late pregnancy compared to non-pregnant mice (Figure 6B). However, no difference was detected in collagen type VI, alpha 3 protein expression between non-pregnant and early pregnant mice cervix, according to proteomics analysis (Figure 6).

\section{Discussion}

This study was designed to decipher the proteome-wide expression of the signature protein in the normal cervix of mice during early and late pregnancy compared to baseline (non- pregnant mice) using proteomics. We show here that various factors associated with cervical

A
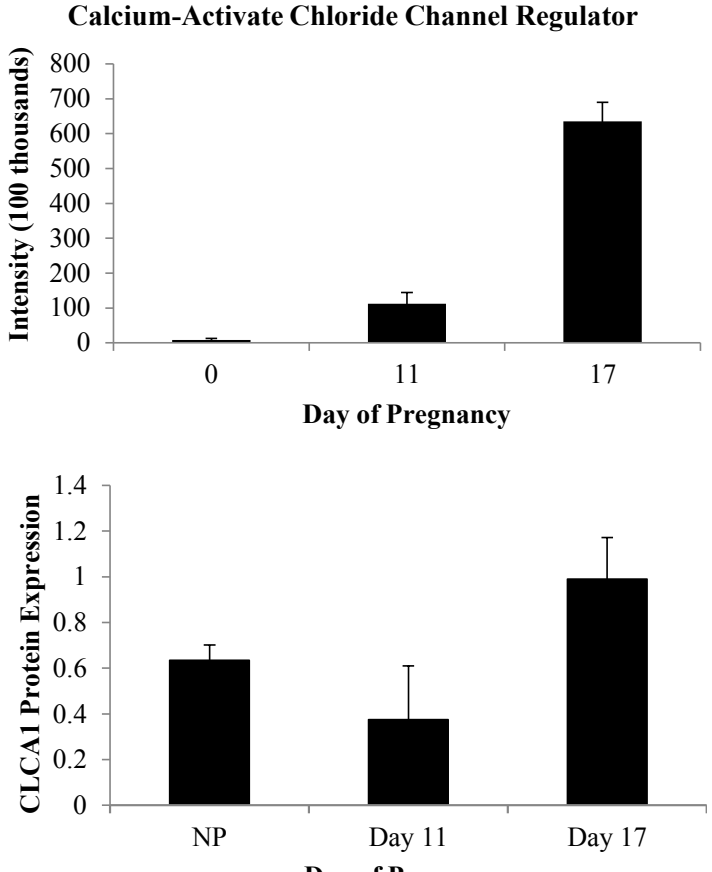

Day of Pregnancy

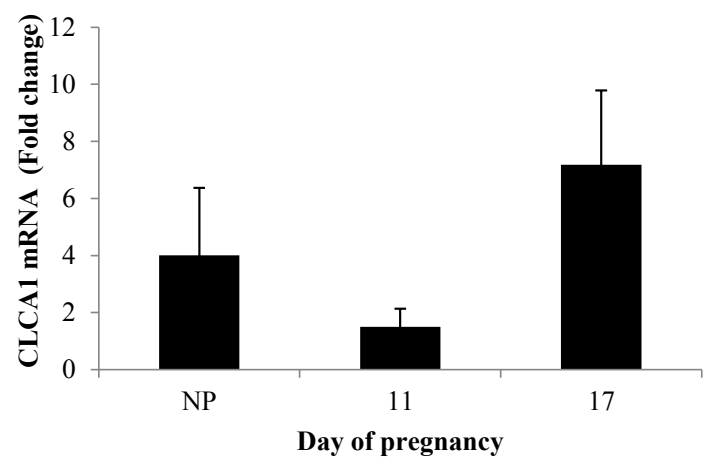

Figure 4: Calcium-Activated Chloride Channel Regulator (CLCAI) levels increase over pregnancy (early vs. late) in mice cervix, as revealed by proteomics (A), and verified by Western blot (B) and real time PCR (C). However, CLCAI mRNA levels in non-pregnant state were higher than early pregnancy and no difference was noted in Western blot analysis. ( $n=3$; and 5 for proteomic and Western blot/real time PCR analyses, respectively; $p<0.05$ for Figure 3B, C: $\mathrm{NP} /$ day 11 vs. day 17 and $3 \mathrm{~A}$ : NP vs. day $11 / 17$ and day 11 vs. day 17 ). 


\section{Anterior Gradient Protein 2}
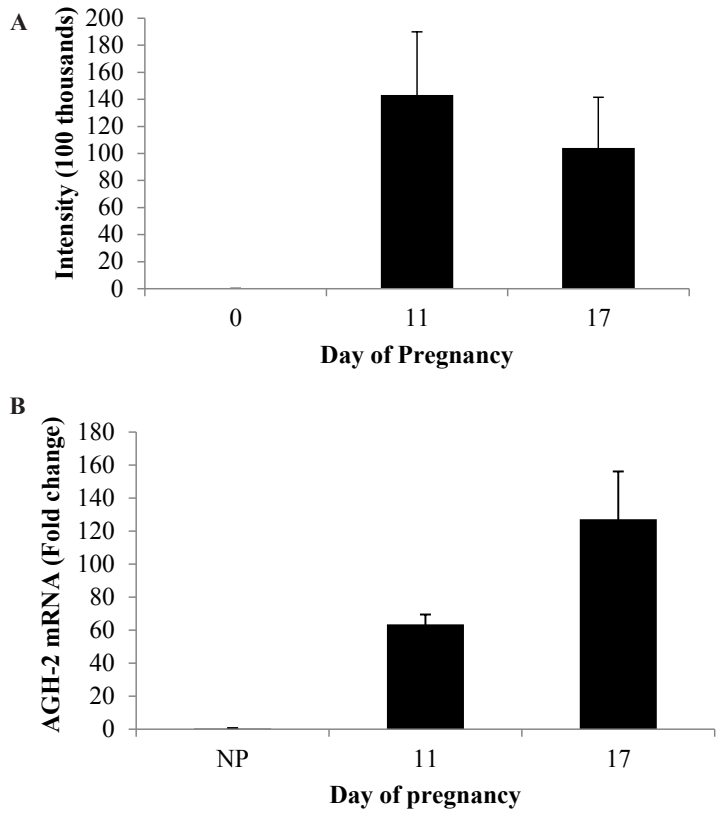

Figure 5: Anterior Gradient Protein 2 (AGH-2) protein and mRNA levels increase in mice cervix during pregnancy compared to non-pregnancy, as revealed by proteomics (A) and confirmed by real time PCR (B). No difference was noted in AGH-2 protein levels between early (day 11) and late (d 17) pregnancy (A). However, mRNA levels were lower in early compared to late pregnancy (B). $(n=3$ and 5 for proteomic and real time PCR analyses, respectively; $p<0.05$ for NP vs. day 11/day 17; Figure 4B: NP vs. day $11 / 17$ and day 11 vs. day 17 ).
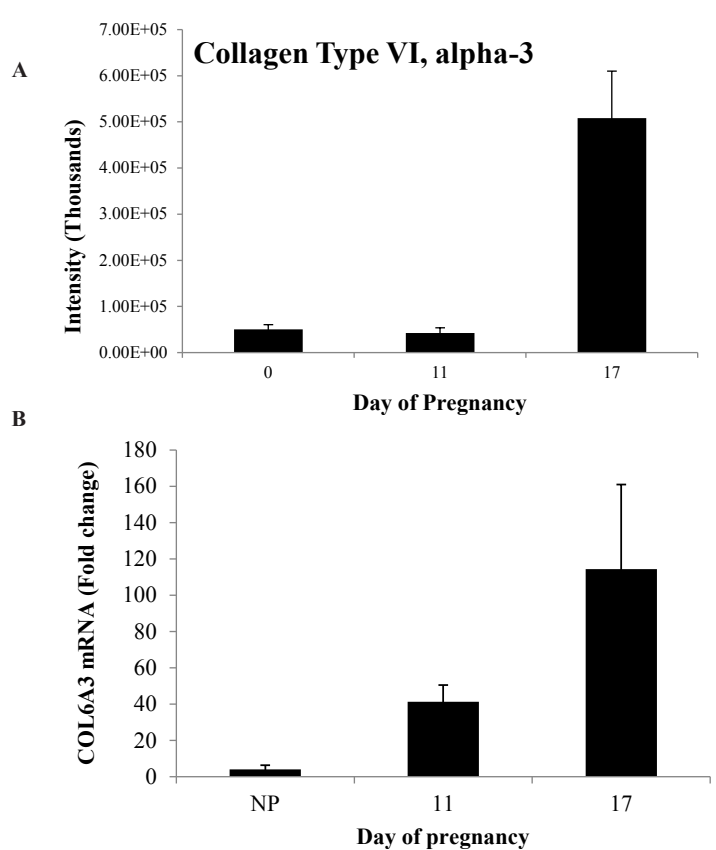

Figure 6: Collagen Type Vi, alpha-3 (COL6A3) mRNA and protein were up regulated in mice cervix during late pregnancy, as revealed by proteomics $(A)$ and confirmed by real time PCR $(B)$. Levels of mRNA in early pregnancy were also found to be higher than non-pregnancy $(B)$. However, no difference was observed in protein expression $(A)$. $(n=3$ and 5 for proteomic and real time PCR analyses, respectively; $p<0.05$ for Figure 5A: NP/day 11 vs. day 17; Figure 5B: NP vs. day $11 / 17$ and day 11 vs. day 17$)$.
A

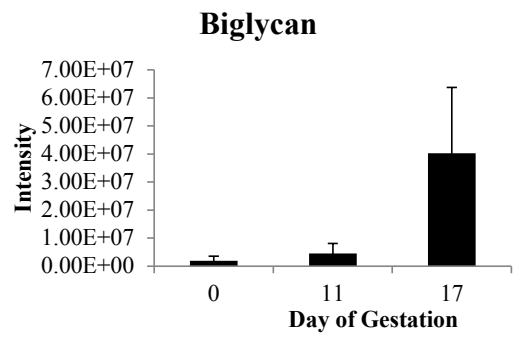

B

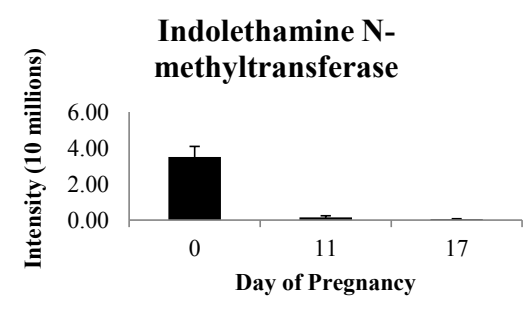

C

Lumican

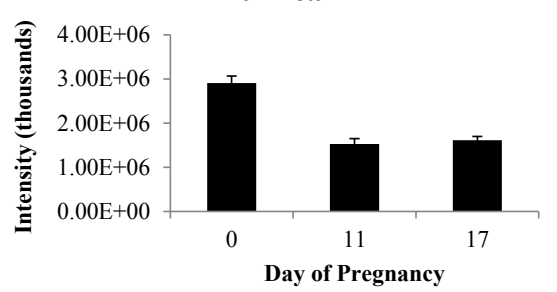

D

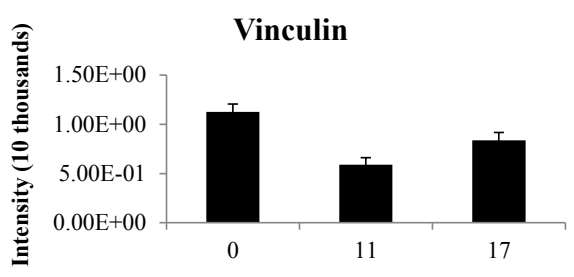

Day of Pregnancy

Figure 7: Proteomic profile of various factors in cervices of non- and pregnant (early and late pregnancy) mice: A: Biglycan protein levels are significantly up-regulated in late pregnancy compared to non- and early- pregnancy $(n=3$, $\mathrm{p}=0.05$, NP/day 11 vs. day 17); B: Indolethanmine N-methyltransferase protein levels are significantly diminished in during pregnancy in general compared to non-pregnancy $(n=3, p=0.05$, NP vs. day 11/17); C: Levels of lumican protein decrease by about $\times 2$ folds in during pregnancy compared to non-pregnancy $(n=3, p=0.05$, NP vs. day 11/17); D: Vinculin protein expression increases over pregnancy (early vs. late), but are lower than in cervix of non-pregnant mice $(n=3, p=0.05$, NP vs. day 11 ; day 11 VS. day 17$)$

epithelia are variably expressed during pregnancy, such as immune, ECF and cytoskeletal factors. The present study adds credence to the recently proposed epithelia model of cervical modeling, both through the variable expression of specific cervical proteins and, overall, through the expression patterns of the different biological themes.

Generally, the most significantly and variably expressed proteins in the cervix in normal pregnancy were those involved in immune regulation and maintenance, cellular adhesion, the cytoskeleton and the key extracellular matrix factors. The immune system has been implicated in cervical remodeling for decades. The Liggins lab was the first to speculate in the 80 s that cervical remodeling is essentially a 


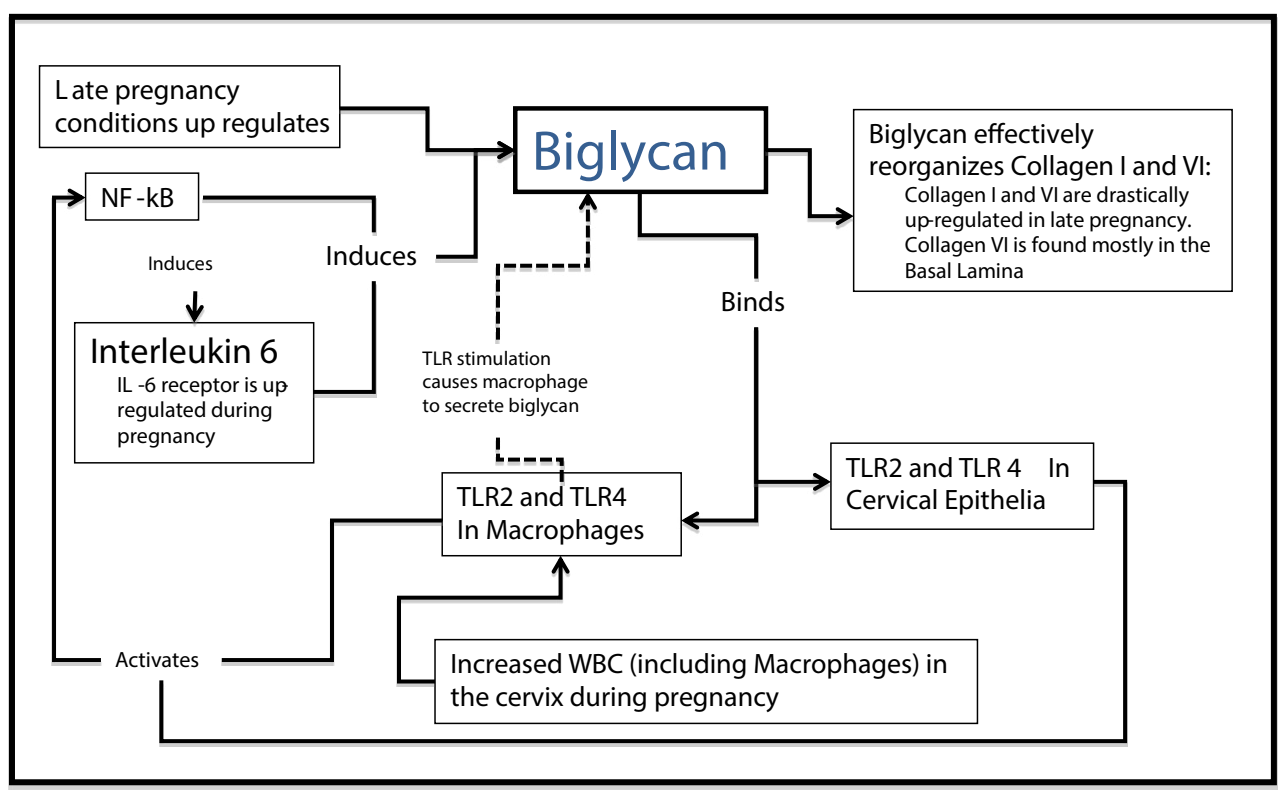

Figure 8: A novel proposed role and working model for biglycan. Biglycan provides a link between the natural- and inflammation-mediated cervical remodeling via its actions involving reorganizing collagen in the ECM and activating pro-inflammatory factors through TLR's and NF- $\mathrm{KB}$.

\begin{tabular}{|c|c|c|}
\hline $\begin{array}{c}\text { Protein functional } \\
\text { biological theme }\end{array}$ & $\begin{array}{c}\text { Number of proteins } \\
\text { changing }\end{array}$ & $\begin{array}{c}\text { Trend of protein } \\
\text { expression in pregnancy }\end{array}$ \\
\hline Protein kinase activity & 2 & Up regulated \\
\hline Proliferation & 9 & Up regulated \\
\hline Calcium binding & 3 & Up regulated \\
\hline Tissue remodeling & 4 & Down regulated \\
\hline Circulation & 3 & Down regulated \\
\hline Immune-related proteins & 11 & Up regulated \\
\hline Nucleus-related proteins & 3 & Up regulated \\
\hline Cellular adhesion & 3 & Up regulated \\
\hline Cytoskeleton-related & 17 & Up regulated \\
\hline proteins & 3 & Down regulated \\
\hline Carbohydrate metabolism & 6 & Up regulated \\
\hline Extracellular matrix & 1 & Up regulated \\
\hline Heat shock proteins & & \\
\hline
\end{tabular}

Table 2: The biological themes are variably expressed in mice cervix during pregnancy (early and late) and in non-pregnant mice. Proteins were assigned to these groups based on function.

physiological inflammatory-like response. It was proposed at that time that white blood cells infiltrate the cervix and secrete inflammatory factors and proteases that breakdown collagen and induce cervical remodeling, notably cervical softening and ripening [8], although that concept was challenged earlier on $[9,10]$. Other factors, such as mucous, secreted by the cervix, provide both a mechanical and chemical barrier against intrauterine infection [20]. Here, we identify eleven variably expressed proteins in mice cervices that are related to immune function, of which mucin $5 \mathrm{~b}$ and calcium-activated chloride channel receptor were the most significantly up regulated. It is interesting to note that these two proteins, i.e., mucin $5 \mathrm{~b}$ and calcium-activated chloride channel receptor, both play an important role in enacting the barrier (mucus plug) that prevents intrauterine microbial invasion. Mucins have also been shown to have similar immune functions in other "border" tissues, such as the bronchial epithelia [21,22]. These finding are consistent with previous proteomics studies on cervical mucus plug that suggested a role for the cervix as the first line of defense in infection and as an immune scavenger [23].
On the contrary, we also show here that a number of proteins that are associated with or promote inflammation and white blood cell activity were slightly down regulated in the cervix during pregnancy compared to non-pregnant mice. Specifically, these proteins include: a) macrophage migration inhibitory factor, which suppresses the antiinflammatory effects of glucocorticoids; b) galectin-1, which is secreted by epithelial cells and indirectly suppresses $\mathrm{T}$ cell function by inducing expression of IL-10 and IL-27; and c) elongation Factor 1-alpha, which increases T cell activity [24-27]. Collectively, these findings do not lend support to earlier suggestions of increased white blood cell activity during pre-softening (day 11) and ripening cervix (day 17), as proposed previously. Instead, it shows a possible involvement of epithelial activity since in other tissue types, each of these altered proteins are largely produced by epithelial cells, i.e., macrophage migration factor 1 in the bladder and major pelvic ganglia; galectin- 1 in the thymus, thyroid and gut; and elongation factor- 1 alpha in the ovaries, trachea and mammary glands [28-31]. Such observation and conclusion are consistent with recent reports that suggest that the cervical epithelia create an immune barrier that protects the uterine cavity from microbial invasion and infiltration during pregnancy [23]. Comprehensive studies on cellular immune-localization and bioassay activity will be essential in resolving this issue.

The largest group of proteins identified in the present study was the cytoskeleton-related proteins. This cluster contains 17 mildly upregulated proteins (fold changes of less than 3), almost all of which bind or associate with actin. Such an expression profile or trend in this cluster of proteins (cytoskeleton and actin binding proteins) is novel in cervical remodeling studies, and as such, their specific roles in cervical remodeling are currently unclear. However, based on their key functions, i.e., proliferation and movement, and their role in mechanotransduction, we can speculate that they are likely involved in cervical growth, which is a prominent feature induced by relaxin during cervical remodelling [16,32]. Interestingly, we have recently shown that VEGF also stimulates genome-wide expression of proliferation- and adhesionrelated genes and induces marked growth of cervical epithelial cells $[18,19]$ and, in other tissues, it (VEGF) has been shown to modulate 
the adhesion and key set of cytoskeletal regulatory molecules, the Rho GTPases, and ultimately, cytoskeletal growth and activity. These molecules (Rho GTPases) are known to direct several steps in morphogenesis, which notably include epithelial cell (EC) motility, alignment, multi-cellular organization, as well as intercellular junction integrity $[33,34]$. The possible involvement of relaxin and VEGF in regulating cytoskeletons and proliferation in the cervix and their collective impact on cervical remodeling will be worth investigating in the future. Vinculin, which was increased in the cervices of mice in late gestation compared to early pregnancy, is a component of cell-cell and cell-matrix adhesions and is an important scaffold protein involved in mechano-transduction pathways [35-37]. Cervical growth in pregnancy is influenced by increased water as well, which also affects the mechanical forces on this tissue [38]. Therefore, the effect of mechanical forces on cervical biochemical changes is also ripe for study. Of note, we identify here a cluster of extracellular matrix proteins that play a critical role in maintaining the biomechanical integrity of the cervix, as well as cervical remodeling, in general. As expected, proteins in this cluster were robustly up regulated during pregnancy, with an average fold change of +29.87 between non-pregnant and early pregnancy states and a similar trend between early and late pregnancy, suggesting an important function for this group in maintaining cervical remodeling during normal pregnancy. For instance, laminin, a protein found mainly in the basal lamina surrounding blood vessels and separating the epithelial cells, was variably expressed during pregnancy, including two types of collagen (Collagen I and VI) [39]. While collagen I is the primary type of collagen present in the cervix, collagen VI, whose different chains accounts for three of the eight proteins in the ECM group, has not been previously reported in the cervix [10]. This collagen type (VI) is primarily found in the basal lamina and, of relevance to the present study, is efficiently bound by short leucine-rich proteoglycans (SLRP's) [40,41]. Of the total eight proteins identified in the ECM cluster, two are SLRP's, i.e., biglycan and lumican, both of which can bind and reorganize collagen VI and I into fibrils [40,42]. However, while biglycan was found to be significantly up regulated during pregnancy, lumican was, in contrast, down regulated, suggesting that biglycan may play an important role in manipulating cervical collagen during pregnancy. Such a speculation is consistent with previous data that have shown biglycan to have pronounced influence on collagen I and VI reorganization and that collagen fibrils become more organized later in pregnancy [17]. Further, it is likely that biglycan and, perhaps decorin $[18,43]$, may be involved in collagen reorganization into large, spaced out fibrils that lead to enhanced cervical softening, and that this process could be facilitated by a decrease in the expression of lysyl oxidase, the enzyme that is known to cross-link collagen molecules and create a dense network of thin collagen fibrils [1].

While biglycan is known to be an efficient regulator of collagen organization, in some tissues, it has also been implicated in inflammatory responses [44]. If such a dual role holds true or exists in the cervix, it is feasible to speculate that biglycan and other molecules with similar dual effects, such as VEGF $[18,45]$, may provide the link between the abnormal inflammation- related and normal or physiological cervical remodeling, through their involvement in both ECM organization and inflammation. Interestingly, biglycan is also necessary for pregnancy to be carried to term in mice [46]. Specifically, biglycan enhances inflammation by binding to and activating toll-like receptors 2 and 4 (TLR2, TLR4), which are receptors for lipopolysaccharide (LPS), the endotoxin of Gram-negative bacteria located in the outer membrane, and responsible for eliciting strong immune responses. These receptors are expressed in both cervical epithelia and macrophages, cell types that notably are present in significant numbers in the cervix during pregnant [44-49]. The TLR molecules, in turn, activate the master transcription factor of inflammation, namely $\mathrm{NF \kappa B}$, which induces the transcription of many classical pro- inflammatory genes. Importantly, biglycan production is also known to be induced by activated

NF- $\kappa B$ signaling pathway and by IL- 6 , a pro-inflammatory cytokine downstream of NF- $\mathrm{KB}$ [49], suggesting a likely existence of a positivefeed forward mechanism in extra-cervical tissues. If such a loop exists in the remodeling cervix, it may possibly help explain the link between the two competing models of cervical remodeling, i.e., the expression of biglycan in normal cervical remodeling is up regulated, leading to the reorganization of collagen in the ECM of the cervix. Whereas, during infection-mediated cervical remodeling, increased levels of biglycan enhances inflammation by activating TLR and molecules downstream of it, such as NFKB and pro-inflammatory factors. Preliminary data from on-going studies in our lab support this alternative model. However, it is important to note that more work is required to rigorously test the proposed model.

It is important to note that the 206 proteins identified here are, without a doubt, far less than the actual total number of proteins expressed in the cervix during pregnancy, perhaps reflecting the limitations in the sensitivity of the LC/MS/MS procedure of labelfree proteomics compared to other genome-wide techniques. It is also possible that expression of the less abundant proteins were overshadowed by those of the dominant fibrous elements of the cervix, which are about $80 \%$ of the tissue. This is the major limitation of the present study. Secondly, there were a few instances when trends in the proteomic data were not perfectly verified by Western and real time data. A recent review by Vogel and Marcotte (2012) provides insights into the correlation of proteomics, gene and protein expression, and states that these correlations can be as little as $40 \%$, depending on the system [50]. Lastly, as in all previously published studies in this field, it is possible that vaginal and uterine tissues, as well as blood components, may not have been excluded completely during cervical tissue harvest, even though great care was taken to prevent tissue contamination during this process.

In conclusion, we show here that cervical epithelial-associated factors, such as those involved in immune regulation, ECM organization and cytoskeletal activities, are active during normal pregnancy, suggesting a role for cervical epithelia in normal cervical remodeling. Additionally, we propose a new model and additional role for biglycan in the cervix, i.e., it may provide the link between the pathological inflammation-induced and the physiological cervical remodeling (Figure 8). This model remains to be tested.

\section{Acknowledgement}

This study was funded by Appalachian State University Office of Student Research and the College of Arts and Sciences.

\section{References}

1. Timmons B, Akins $M$, Mahendroo $M$ (2010) Cervical remodeling during pregnancy and parturition. Trends Endocrinol Metab 21: 353-361.

2. Read CP, Word RA, Ruscheinsky MA, Timmons BC, Mahendroo MS (2007) Cervical remodeling during pregnancy and parturition: molecular characterization of the softening phase in mice. Reproduction 134: 327-340.

3. Word RA, Li XH, Hnat M, Carrick K (2007) Dynamics of cervical remodeling during pregnancy and parturition: mechanisms and current concepts. Semin Reprod Med 25: 69-79.

4. Stencel C (2006) Preterm Births Cost U.S. \$26 Billion a Year; Multidisciplinary Research Effort Needed to Prevent Early Births. In. Washington D.C.: The National Academies. 
Citation: Schwabe J, Donnelly SM, Jesmin S, Leppert P, Mowa CN (2014) A Proteomic Profile of Cervical Remodeling in Mice during Early and Late Pregnancy. J Steroids Horm Sci 5: 123. doi:10.4172/2157-7536.1000123

5. Blencowe H, Cousens S, Oestergaard MZ, Chou D, Moller AB, et al. (2012) National, regional, and worldwide estimates of preterm birth rates in the year 2010 with time trends since 1990 for selected countries: a systematic analysis and implications. Lancet 379: 2162-2172.

6. Gonzalez JM, Dong Z, Romero R, Girardi G (2011) Cervical remodeling ripening at term and preterm delivery: the same mechanism initiated by different mediators and different effector cells. PLoS One 6: e26877.

7. Gonzalez JM, Franzke CW, Yang F, Romero R, Girardi G (2011) Complement activation triggers metalloproteinases release inducing cervical remodeling and preterm birth in mice. Am J Pathol 179: 838-849

8. Liggins GC (1981) Cervical ripening as an inflammatory reaction. In The Cervix in Pregnancy and Labour, Clinical and Biochemical Investigation, Ellwood D \& Anderson ABM. Edinburgh: Churchill Livingston.

9. Leppert PC (1992) Cervical softening, effacement and dilatation: a complex biochemical cascade. J Materal Fetal Medicine 1: 213-223.

10. Leppert PC (1995) Anatomy and physiology of cervical ripening. Clin Obste Gynecol 38: 267-279.

11. Huck O, Tenenbaum H, Davideau JL (2011) Relationship between periodonta diseases and preterm birth: recent epidemiological and biological data. $J$ Pregnancy 2011: 164654.

12. Adams Waldorf KM, Rubens CE, Gravett MG (2011) Use of nonhuman primate models to investigate mechanisms of infection-associated preterm birth. BJOG 118: $136-144$.

13. Kemp MW, Saito M, Newnham JP, Nitsos I, Okamura K, et al. (2010) Preterm birth, infection, and inflammation advances from the study of animal models. Reprod Sci 17: 619-628.

14. Nold C, Anton L, Brown A, Elovitz M (2012) Inflammation promotes a cytokine response and disrupts the cervical epithelial barrier: a possible mechanism of premature cervical remodeling and preterm birth. Am J Obstet Gynecol 206 208.

15. Holt R, Timmons BC, Akgul Y, Akins ML, Mahendroo M (2011) The molecular mechanisms of cervical ripening differ between term and preterm birth. Endocrinology 152: 1036-1046

16. Timmons BC, Mahendroo MS (2006) Timing of neutrophil activation and expression of proinflammatory markers do not support a role for neutrophils in cervical ripening in the mouse. Biol Reprod 74: 236-245.

17. Mahendroo $M$ (2012) Cervical remodeling in term and preterm birth: insights from an animal model. Reproduction 143: 429-438.

18. Mowa CN, Li T, Jesmin S, Folkesson HG, Usip SE, et al. (2008) Delineation of VEGF-regulated genes and functions in the cervix of pregnant rodents by DNA microarray analysis. Reprod Biol Endocrinol 6: 64

19. Donnelly SM, Nguyen BT, Rhyne S, Estes J, Jesmin S, et al. (2013) Vascular endothelial growth factor induces growth of uterine cervix and immune cel recruitment in mice. J Endocrinol 217: 83-94.

20. Ming L, Xiaoling P, Yan L, Lili W, Qi W, et al. (2007) Purification of antimicrobial factors from human cervical mucus. Hum Reprod 22: 1810-1815.

21. Buckner LR, Schust DJ, Ding J, Nagamatsu T, Beatty W, et al. (2011) Innate immune mediator profiles and their regulation in a novel polarized immortalized epithelial cell model derived from human endocervix. J Reprod Immunol 92:

22. Kim KC (2012) Role of epithelial mucins during airway infection. Pulm Pharmacol Ther 25: 415-419.

23. Lee DC, Hassan SS, Romero R, Tarca AL, Bhatti G, et al. (2011) Protein profiling underscores immunological functions of uterine cervical mucus plug in human pregnancy. J Proteomics 74: 817-828.

24. Cedeno-Laurent F, Dimitroff CJ (2012) Galectin-1 research in T cell immunity: past, present and future. Clin Immunol 142: 107-116.

25. Cedeno-Laurent F, Opperman M, Barthel SR, Kuchroo VK, Dimitroff CJ (2012) Galectin-1 triggers an immunoregulatory signature in Th cells functionally defined by IL-10 expression. J Immunol 188: 3127-3137.

26. Flaster H, Bernhagen J, Calandra T, Bucala R (2007) The macrophage migration inhibitory factor-glucocorticoid dyad: regulation of inflammation and immunity. Mol Endocrinol 21: 1267-1280.
27. Yan G, You B, Chen SP, Liao JK, Sun J (2008) Tumor necrosis factor-alpha downregulates endothelial nitric oxide synthase mRNA stability via translation elongation factor 1-alpha 1. Circ Res 103: 591-597.

28. Ann DK, Wu MM, Huang T, Carlson DM, Wu R (1988) Retinol-regulated gene expression in human tracheobronchial epithelial cells. Enhanced expression of elongation factor EF-1 alpha. J Biol Chem 263: 3546-3549.

29. Perillo NL, Uittenbogaart CH, Nguyen JT, Baum LG (1997) Galectin-1, an endogenous lectin produced by thymic epithelial cells, induces apoptosis of human thymocytes. J Exp Med 185: 1851-1858.

30. Kolettas E, Lymboura M, Khazaie K, Luqmani Y (1998) Modulation of elongation factor-1 delta (EF-1 delta) expression by oncogenes in human epithelial cells. Anticancer Res 18: 385-392.

31. Vera PL, Meyer-Siegler KL (2003) Anatomical location of macrophage migration inhibitory factor in urogenital tissues, peripheral ganglia and lumbosacral spinal cord of the rat. BMC Neurosci 4: 17

32. Sherwood OD (2004) Relaxin's physiological roles and other diverse actions. Endocr Rev 25: 205-234.

33. Spencer KSR and Cheresh DA (2002) VEGF-Induced Cytoskeletal Alteration Relating to Vascular Permeability in Endothelial Cells. Microscopy and Microanalysis 8 Supplement S02: 1046-1047.

34. Nagy JA, Senger DR (2006) VEGF-A, cytoskeletal dynamics, and the pathological vascular phenotype. Exp Cell Res 312: 538-548.

35. Leerberg JM, Yap AS (2013) Vinculin, cadherin mechanotransduction and homeostasis of cell-cell junctions. Protoplasma 250: 817-829.

36. Goldmann WH, Auernheimer V, Thievessen I, Fabry B (2013) Vinculin, cel mechanics and tumour cell invasion. Cell Biol Int 37: 397-405.

37. Thompson PM, Tolbert CE, Campbell SL (2013) Vinculin and metavinculin: oligomerization and interactions with F-actin. FEBS Lett 587: 1220-1229.

38. Drzewiecki G, Tozzi C, Yu SY, Leppert PC (2005) A Dual Mechanism of Biomechanical Change in Rat Cervix in Gestation and Postpartum: Applied Vascular Mechanics. Cardiovascular Engineering 5: 187-193.

39. Timpl R, Rohde H, Robey PG, Rennard SI, Foidart JM, et al. (1979) Laminin--a glycoprotein from basement membranes. J Biol Chem 254: 9933-9937.

40. Wiberg C, Heinegård D, Wenglén C, Timpl R, Mörgelin M (2002) Biglycan organizes collagen $\mathrm{VI}$ into hexagonal-like networks resembling tissue structures. J Biol Chem 277: 49120-49126.

41. Sjöberg AP, Manderson GA, Mörgelin M, Day AJ, Heinegård D, et al. (2009) Short leucine-rich glycoproteins of the extracellular matrix display diverse patterns of complement interaction and activation. Mol Immunol 46: 830-839.

42. Chakravarti S (2002) Functions of lumican and fibromodulin: lessons from knockout mice. Glycoconj J 19: 287-293.

43. Leppert PC, Kokenyesi R, Klemenich CA, Fisher J (2000) Further evidence of a decorin-collagen interaction in the disruption of cervical collagen fibers during rat gestation. Am J Obstet Gynecol 182: 805-811.

44. Babelova A, Moreth K, Tsalastra-Greul W, Zeng-Brouwers J, Eickelberg O, et al. (2009) Biglycan, a danger signal that activates the NLRP3 inflammasome via toll-like and P2X receptors. J Biol Chem 284: 24035-24048.

45. Nguyen BT, Minkiewicz V, McCabe E, Cecile J, Mowa CN (2012) Vascular endothelial growth factor induces mRNA expression of pro-inflammatory factors in the uterine cervix of mice. Biomed Res 33: 363-372.

46. Wu Z, Aron AW, Macksoud EE, lozzo RV, Hai CM, et al. (2012) Uterine dysfunction in biglycan and decorin deficient mice leads to dystocia during parturition. PLoS One 7: e29627.

47. Ungefroren H, Krull NB (1996) Transcriptional regulation of the human biglycan gene. J Biol Chem 271: 15787-15795

48. Pioli PA, Amiel E, Schaefer TM, Connolly JE, Wira CR, et al. (2004) Differentia expression of Toll-like receptors 2 and 4 in tissues of the human female reproductive tract. Infect Immun 72: 5799-5806.

49. Schaefer L, Babelova A, Kiss E, Hausser HJ, Baliova M, et al. (2005) The matrix component biglycan is proinflammatory and signals through Toll-like receptors 4 and 2 in macrophages. J Clin Invest 115: 2223-2233.

50. Vogel C, Marcotte EM (2012) Insights into the regulation of protein abundance from proteomic and transcriptomic analyses. Nat Rev Genet 13: 227-232. 